Enferm Bras 2019;18(6):767-74

https://doi.org/10.33233/eb.v18i6.3242

\title{
ARTIGO ORIGINAL \\ Fórum de discussão online sobre hipertensão arterial sistêmica na perspectiva da teoria da cognição situada
}

Flávia de Oliveira, D.Sc. ${ }^{*}$ Gláucia Daniele Pereira Assis ${ }^{\star *}$, Marlene das Graças Martins, M.Sc. ${ }^{* \star *}$, Marcos de Abreu Nery, D.Sc. ${ }^{* \star *}$, Sueli Leiko Takamatsu Goyata, D.Sc. ${ }^{* \star \star \star *}$

${ }^{*}$ Professora Adjunta da Universidade Federal de São João Del Rei/MG, ${ }^{* \star}$ Especialista em Atenção Básica/Saúde da Família, Universidade Federal de São João Del Rei/MG, ${ }^{* * *}$ Universidade Federal de Alfenas, Alfenas/MG, ${ }^{* * * *}$ Técnico de Assunto Educacionais da Universidade Federal de Alfenas, Alfenas/MG, ${ }^{* * * \star *}$ Professora Adjunta da Universidade Federal de Alfenas, Alfenas/MG

Recebido em 23 de setembro de 2019; aceito em 10 de dezembro de 2019.

Correspondência: Flávia de Oliveira, Universidade Federal de São João Del Rei, Rua Sebastião Gonçalves Coelho, 400, Chanadour, Bloco D, sala 303.4, Campus Centro-Oeste Dona Lindu 35501-296 Divinópolis/MG

Flávia de Oliveira: flaviadeoliveira@ufsj.edu.br

Gláucia Daniele Pereira Assis: gpereiraassis@gmail.com

Marlene das Graças Martins: marlenemartinspaz@yahoo.com.br

Marcos de Abreu Nery: marcosnery2009@gmail.com

Sueli Leiko Takamatsu Goyata: sueligoyata@yahoo.com.br

\section{Resumo}

Introdução: A Teoria da Cognição Situada destaca o potencial do trabalho em grupo e é significativa para a compreensão do acesso ao conhecimento e à criatividade. Portanto, propõese que essa teoria possa fundamentar as bases teóricas do processo de ensino-aprendizagem on-line na área da saúde. Objetivo: Relatar a experiência da utilização de um fórum de discussão online sobre hipertensão arterial sistêmica a partir da teoria da cognição situada. Métodos: Participaram do estudo 20 discentes dos cursos de enfermagem, farmácia, fisioterapia, nutrição e odontologia da Universidade Federal de Alfenas. Foi criado um fórum de discussão online, inserido em um curso oferecido pela Pró-reitora de Extensão da UNIFAL-MG, sobre o tema Hipertensão Arterial Sistêmica na plataforma moodle. A teoria da cognição situada foi o referencial teórico que orientou a elaboração da pesquisa em conjunto com o recurso in Map Moodle. Resultados: Foi possível observar que as colocações estavam correlacionadas a prática social vivenciada, o engajamento dos participantes ocorreu de forma processual, resultando em um processo de compartilhamento social, e a multidisciplinaridade presente no fórum auxiliou na construção social para propor soluções para o caso apresentado no fórum. Conclusão: A Teoria da Cognição Situada mostrou consistente para o processo de ensino-aprendizagem proposto neste estudo por considerar o aluno como um ser social interativo.

Palavras-chave: fóruns de discussão, aprendizagem colaborativa, educação a distância, atenção integral a saúde.

\footnotetext{
Abstract

Online discussion forum on systemic arterial hypertension in the perspective of cognition theory

Introduction: The situation Cognition Theory detaches the potential of group work and it is significant for understand the access of knowledge and creativity. Therefore, it is proposed that this theory may justify the theoretical foundations of the online teaching-learning process in healthy. Objective: To report the experience of using an online discussion forum on systemic arterial hypertension based on the theory of situated cognition. Methods: Twenty students from nursing, pharmacy, physical therapy, nutrition and dentistry courses of the Federal University of Alfenas participated in the study. An online discussion online was created, inserted in a course offered by the Extension Dean of Unifal/MG, on the theme Systemic Arterial Hypertension on the moodle platform. The situated cognition theory was the theoretical referential that guided the
} 
elaboration of the research in conjunction with the Map Moodle resource. Results: It was possible to notice that the statements were correlated to the social practice experienced, the participants' engagement occurred procedurally, resulting in a process of social sharing, and the multidisciplinarity present in the forum helped in the social construction to propose solutions for the case presented in the forum. Conclusion: The Cognition Theory situation showed to be consisted for the teaching-learning process proposed in this study because it considers the student as an interactive social being.

Key-words: discussion forums, collaborative learning, distance education course, comprehensive healthcare.

\section{Resumen \\ Foro de discusión en red acerca de hipertensión arterial sistémica en la perspectiva de la teoría cognición situada \\ Introducción: La teoría de la Cognición situada subraya el potencial del trabajo en equipo y es significativa para la comprensión del acceso al conocimiento y a la creatividad. Entonces, se propone que esa teoría pueda fundamentar las bases teóricas del proceso de enseñanza- aprendizaje en red acerca de la hipertensión arterial sistémica además de la teoría de cognición situada. Métodos: Participaran del equipo 20 discentes de cursos de enfermería, farmacia, fisioterapia, nutrición y odontología de la Universidad Federal de Alfenas. Fue creado un foro de discusión en red, inserido en un curso ofrecido por la Pro-rectoría de Extensión de la Unifal/MG, acerca del tema Hipertensión Arterial Sistémica en la plataforma digital Moodle. La teoría de cognición situada fue referencial teórico que apuntó la elaboración de la encuesta en conjunto con el recurso in Map Moodle. Resultados: Fue posible mirar que las colocaciones estaban correlacionadas a la práctica social vivida, la aproximación de los participantes ocurrió de forma procesual, resultando en un proceso de compartimiento social, y la multidisciplinaria presente en el foro, ayudó en la construcción social para proponer soluciones conscientes para el proceso del foro. Conclusión: La teoría de la cognición situada mostró consistente para el proceso de enseñanza-aprendizaje propuesto en este estudio por considerar el alumno como un ser social interactivo.}

Palabras-clave: foro de discusión, aprendizaje colaborativo, educación a la distancia, atención integral a la salud.

Introdução

Historicamente, os métodos tradicionais de ensino e aprendizagem sempre estiveram ancorados na transmissão de conhecimentos. Em contraposição, na atualidade, surgem algumas abordagens cognitivas, entre elas o arcabouço teórico conceitual que vem sendo denominado de Teoria da Cognição Situada.

A cognição é tida como um fenômeno social complexo no qual a atividade cognitiva é situada em função das relações que se estabelecem entre o sujeito que age cognitivamente e o contexto, a cultura, o meio social onde essa ação se desenvolve [1]. Ou seja, não pode estar dissociada da prática social e do objetivo comum que leva às atividades dos participantes.

A abordagem proposta por Clancey [2], busca compreender a cognição a partir de seus aspectos funcionais, estruturais e comportamentais, evidenciando que todo ato cognitivo é um ato experimental e, portando, situado, resultante do acoplamento estrutural e da coevolução congruente do ser vivo e de seu ambiente. Além do mais, a aprendizagem situada combina atividades, compartilhamentos, relacionamentos, cooperação, dialética, interação, negociação, observação, aperfeiçoamento, significado e criatividade [1,2].

Ao considerar esses aspectos é iminente repensar as práticas pedagógicas utilizadas na área da saúde que permaneceram durante décadas centradas na abordagem biologista e procedimental. A prática educacional deve, então, superar as fragmentações entre teoria e prática, a desarticulação entre disciplinas, a descontextualização de saberes, o predomínio do uso de metodologias passivas de ensino-aprendizagem, o privilégio da memorização em detrimento do raciocínio e a abordagem atomística de competência [3]. Para atender essa necessidade, a educação superior necessita considerar estratégias didático-pedagógicas ou modos de ensinar problematizadores.

A utilização das Tecnologias de Informação e Comunicação (TIC) na Educação podem ser capazes de influenciar positivamente o processo de ensino e aprendizagem, pois trazem novas possibilidades na formação profissional [4]. Além do mais, a contextualização das 
aprendizagens, com suportes na filosofia e sociologia da educação e em teorias da aprendizagem, tem sido uma proposta muito aceita atualmente no campo da educação [5].

O ambiente virtual Moodle (Modular Object-Oriented Dynamic Learning Environment), idealizado segundo princípios sócio construtivistas, é um Ambiente Virtual de Aprendizagem de uso livre, que conta com recursos de comunicação síncrona, como chats, e assíncrona, como fóruns de discussão, wikis (textos colaborativos) e blogs [6].

O fórum de discussão, em especial, é uma ferramenta interativa que propicia um verdadeiro contexto dialógico, no qual as trocas se caracterizam não apenas pelo encadeamento das mensagens, mas, por criar um espaço de compartilhamento de experiências e perspectivas, visando o fomento da discussão e o aprofundamento das reflexões a respeito do conteúdo [7].

Sendo assim, propõe-se que a teoria da Cognição Situada possa fundamentar a base teórica do processo de ensino-aprendizagem no Ambiente Virtual de Aprendizagem (AVA), em especial no fórum de discussão, por considerar o usuário como um "ser social" interativo capaz de estabelecer relações e produzir o conhecimento contextualizado em sua realidade prática. Mediante o exposto, o presente estudo teve como objetivo elaborar e analisar um fórum de discussão virtual a luz da teoria da cognição situada.

\section{Material e métodos}

Pesquisa descritiva de análise qualitativa dos dados [8], realizada na plataforma Moodle com auxílio de um técnico educacional e uma tutora à distância da Universidade Federal de Alfenas. O referencial teórico que orientou a criação, o desenho, o processo de interação tutoraluno, a coleta dos dados e a posterior análise, em conjunto com o recurso in Map Moodle, foi a teoria da cognição situada.

Como critério de inclusão utilizou-se: discentes regularmente matriculados nos cursos da área da saúde na Universidade Federal de Alfenas, que aceitassem participar da pesquisa e assinassem o Termo de Consentimento Livre e Esclarecido, como critério de exclusão tem-se os discentes que não atenderam aos critérios de inclusão. Utilizou-se a amostragem probabilística aleatória simples composta por quatro participantes do curso de enfermagem, farmácia, fisioterapia, nutrição e odontologia, totalizando assim, 20 participantes.

Foi elaborado um fórum de discussão online sobre a hipertensão arterial sistêmica inserido em um curso oferecido pela Pró-reitora de Extensão da UNIFAL-MG. O fórum foi mediado por uma tutora com experiência na temática e em educação à distância e norteado pelo referencial da Teoria da Cognição Situada [9], que tem como características principais:

1. Início enraizado em uma atividade familiar legítima do conhecimento prévio dos alunos, servindo de base para estes adentrarem em atividades não-familiares;

2. Demonstração de várias formas para solucionar um problema;

3. Possibilidade de tornar os alunos ativamente envolvidos na cultura ao permitir que eles construam suas próprias abordagens de soluções.

O fórum foi realizado nos meses de fevereiro a março de 2013 sendo dividido em duas partes. Inicialmente foi realizada uma discussão a partir dos conhecimentos prévios dos participantes, mediante a seguinte questão "Porque a Hipertensão Arterial Sistêmica é um problema de saúde coletiva na atualidade?"

Ao finalizar essa primeira discussão, a tutora indicou leituras de referências científicas, e logo após a segunda parte do fórum foi iniciada com o seguinte questionamento "Quais os problemas relacionados aos encaminhamentos da atenção primária para as especialidades?"

Diante dessa reflexão, "quais seriam as implicações desses problemas no atendimento às necessidades na rede de atenção?" Esses questionamentos tiveram como objetivo instigar os participantes para a resolução de problemas e a partir de então propor soluções colaborativamente segundo princípios sócio construtivistas.

Para a análise qualitativa dos dados, as falas foram lidas e analisadas, utilizando-se da análise de conteúdo proposta por Minayo [8]. Foi realizada uma leitura exaustiva de todo o material obtido para possibilitar que a imersão nos dados evidenciasse os temas ou padrões recorrentes que possibilitasse o aprofundamento e a compreensão analítica.

A pesquisa foi aprovada pelo Comitê de Ética e Pesquisa da Universidade Federal de Alfenas em 5 de maio de 2011, sob o Parecer no 010/2011. Todos os participantes assinaram o Termo de Consentimento Livre e Esclarecido baseado e atenderam as Diretrizes e Normas Regulamentadoras de Pesquisa envolvendo seres Humanos. 
O fórum inicialmente apresentou 61 contribuições, no entanto a tutora realizou diversas intervenções a fim de incentivar essa discussão. Além disso, grande parte dos participantes postou seu ponto de vista mais de uma vez. Na segunda parte houve 29 contribuições, em que algumas pessoas que não participaram da primeira fase do fórum postaram respostas nessa segunda fase. Apenas duas participantes não contribuíram em nenhuma das fases do fórum.

No moodle da Universidade Federal de Alfenas existe um recurso denominado in Map Moodle que permite visualizar as interações entre os usuários. $\mathrm{O}$ in Map Moodle foi desenvolvido no ano de 2012 pela Agência de Inovação e Empreendedorismo da universidade, com o objetivo de permitir a visualização das interações em mapas das discussões mediados pelos fóruns, com indicação de setas no sentido das mensagens, utilizando escalas de cores para representar a intensidade da troca de mensagens entre os usuários. Permite ainda a visualização dos fluxos de comunicação nos fóruns de discussão do moodle de modo visual, assim como da quantificação em escalas de cores das mensagens enviadas, possibilitando importantes informações para avaliação educacional dos fóruns.

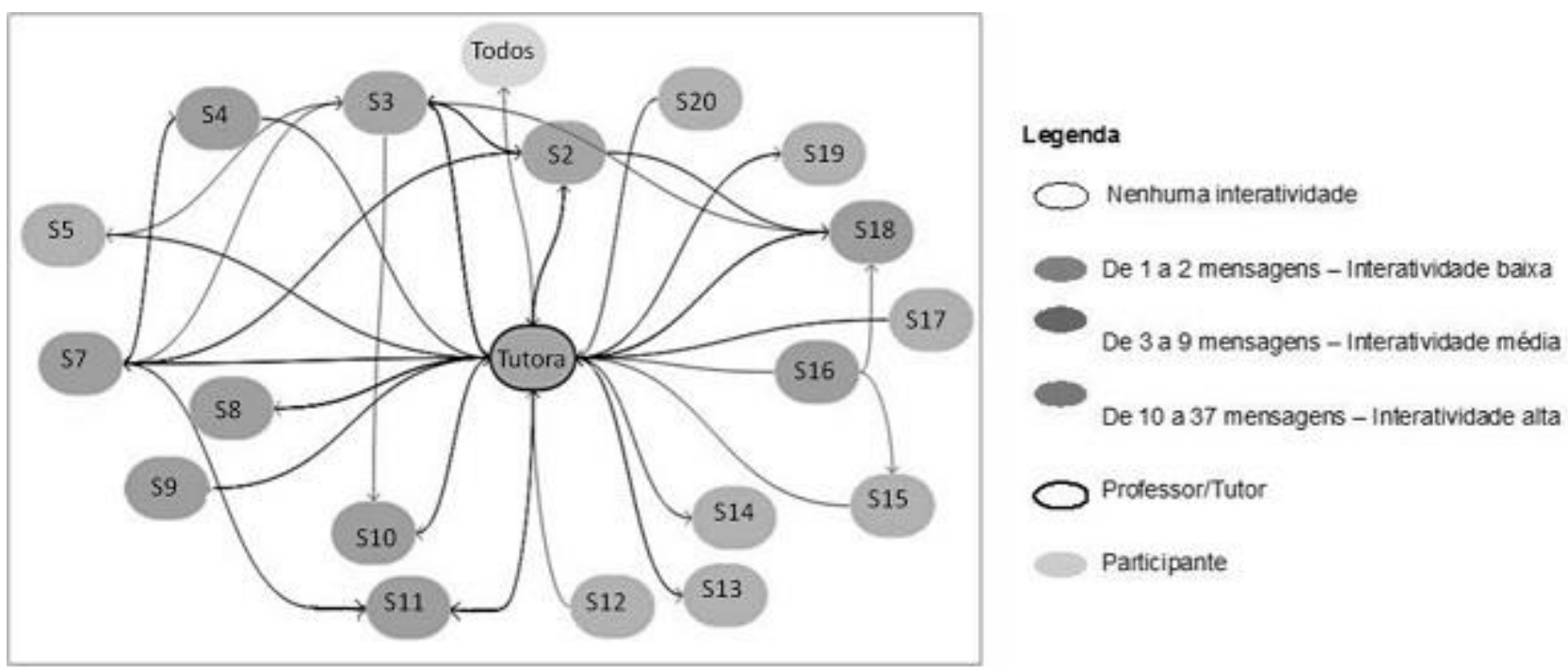

Figura 1 - Mapa de interação do Fórum - Fonte: in Map Moodle UNIFAL-MG.

Por meio desta ferramenta foi possível observar que alguns participantes foram mais ativos na discussão do fórum, enquanto outros apresentaram poucas contribuições. A tutora assumiu o papel de mediadora das discussões e interagiu com todos os participantes. Em relação a interatividade, 10\% tiveram interatividade alta, 40\% tiveram interatividade média, $40 \%$ interatividade baixa e $10 \%$ não participou. É possível identificar que os participantes estabeleceram interação com a tutora, bem como entre os outros participantes.

Em relação às postagens dos participantes, diversas colocações estavam correlacionadas à prática social vivenciada por eles.

Fico me perguntando... E aquelas pessoas sem cobertura de PSF? Que não possuem o acompanhamento diário e contínuo do PSF? Como fazem para manter a doença sob controle? (Participante 5).

Em relação ao processo de compartilhamento social, o mesmo ocorreu de acordo com os vínculos estabelecidos nas discussões. O engajamento dos participantes ocorreu de forma processual. No início das discussões os participantes apenas respondiam as perguntas da tutora. Após o engajamento no grupo, as discussões ocorreram entre os participantes, demonstrando 0 estreitamento das relações na construção do conhecimento, conforme demonstrado pelas postagens:

A participante 10 ressaltou que no PSF o paciente deve ser (se preciso) encaminhado ao especialista que o encaminha (se preciso) ao hospital e o hospital o contra referência para o PSF. Porém cabe lembrar que nos momentos de agudização o PSF deve estar preparado para 
referenciar esse paciente ao hospital e não somente o médico especialista, que na maioria não se encontra em contato frequente com essa população, mas sim os profissionais do PSF... (Participante 8).

Concordo plenamente com você! Mas infelizmente, o que se vê na maioria dos casos, é uma assistência curativa e não preventiva... esse foco tem que ser modificado... (Participante 15).

Concordo com você em relação à dificuldade de acesso dos pacientes que realmente precisam de um especialista. Isso é um grande problema! Por não ter ou ser feito de forma inadequada a classificação de risco, as filas de espera por um especialista só aumentam, e aqueles pacientes que realmente precisam da consulta, muitas vezes não são atendidos (Participante 19).

Discussão

A evasão está presente em todas as modalidades de ensino, seja presencial ou à distância. A tendência de evasão no ensino a distância pode estar correlacionada ao fato de muitos alunos não estarem adequados e preparados para quebrar paradigmas educacionais e por estarem enraizados no modelo tradicional de ensino [10].

Neste estudo, houve evasão do tipo por abandono e com uma porcentagem abaixo dos índices esperados de uma EAD. De acordo com a Associação Brasileira de Educação a Distância [11], a evasão dos cursos em EAD estão cada vez mais próximas daquelas dos cursos presenciais, variando nos cursos totalmente a distância a taxa de 21 a $25 \%$. A falta de tempo para estudar ou participar do curso é apontada pela maioria das instituições como principal motivo para evasão nas diferentes modalidades de EAD pesquisadas. Pensando nessa questão, o período de fechamento do fórum foi prolongado, cada fase do fórum ficou disponível por um período de 15 a 20 dias para participação, fato que pode ter contribuído positivamente para a baixa evasão.

De acordo com a perspectiva adotada neste estudo, a aprendizagem deve estar associada à prática social e apresentar um objetivo comum. Sendo assim, o fórum de discussão buscou direcionar os participantes para a construção sócio interativa a luz da Teoria da Cognição Situada correlacionada a vivência dos participantes. As perguntas realizadas pela tutora estavam direcionadas para a vivência e práticas sociais, e os mesmos deveriam propor soluções conjuntas para o problema.

A aprendizagem no contexto online, tida como uma construção dinâmica e interrelacional, considera que as ações compartilhadas entre 0 aprendiz e o outro mais experiente/tutor possam direcionar uma ação competente e autônoma. $\mathrm{O}$ tutor ao assumir uma postura crítica-reflexiva possibilita ao estudante pensar sobre o processo de aprendizagem e agir em prol da construção do seu próprio conhecimento [12]. Assim o tutor tem o papel de um postulador de problemas para gerar questionamentos relevantes que conduzam o ensino [13]. Durante todo o fórum, o tutor foi o centralizador e direcionador das discussões, estimulando a interatividade para a aprendizagem crítica-reflexiva necessária na resolução de problemas.

A resolução de problemas é uma habilidade cognitiva de ordem superior, que irá agrupar várias funções cognitivas. Essas funções convergem para a análise, compreensão e resolução de situações que apresentam um problema de formato diverso a resolver [14].

$\mathrm{O}$ conhecimento cognitivo tem uma natureza situada uma vez que existem partes relevantes para seu entendimento que estão presentes no contexto de vida. Essas partes, no entanto, são subjacentes à cultura do conhecimento e ao sistema de valores que essa cultura emprega para usar o conhecimento em diferentes situações [9]. Parte da premissa de que o conhecimento é parte e produto da atividade, do contexto e da cultura. Ou seja, é compreender que a cognição por situada está sempre vinculada às atividades, sendo que essas são passíveis de significações, de representações e de sentidos de diversas tipologias e culturas [13].

No estudo, os participantes, através da atividade proposta e da elaboração de sentidos diversos do arcabouço das experiências já vivenciadas, foram atores sociais do seu próprio processo de ensino-aprendizagem, na tentativa de adquirir a competência e habilidade necessária para a resolução do problema proposto. O conhecimento ocorreu concomitantemente ao engajamento ao grupo, à medida que o participante passava a ter o sentimento de pertencimento ao grupo, a troca de conhecimento ocorria não apenas entre aluno-tutor, mas também entre aluno-aluno. 
Dessa forma, para Wenger [15], a aprendizagem é resultado de um processo de compartilhamento social, e é estabelecida por quatro premissas:

1. O homem é um ser social, fato que se constitui e um aspecto central da aprendizagem;

2. O conhecimento é uma questão de competência;

3. Conhecer é questão de participar de empreendimentos ativos de engajamento no mundo;

4. Significado é a capacidade de experimentar o mundo e o compromisso com aprendizado; é tão significativo quanto a capacidade de aprender a produzir.

A Cognição Situada tem como princípio epistemológico fundamental a existência do organismo-em-seu-ambiente, ou seja, o organismo e o ambiente constituem uma unidade inseparável, e a dinâmica ocorre contínua e simultaneamente. A informação é vista como uma possível perturbação da estrutura biológica do ser que pode ou não ser determinada em função da sua estrutura e da história de interações com o meio. O conhecimento, na cognição situada, é dinâmico e construído pelo ser vivo em suas interações com o mundo [16].

O estudo da cognição não pode ser separado do ambiente, no qual a aprendizagem acontece [17]. Nessa perspectiva, o usuário é um ser que vivencia inúmeras experiências pessoais e intransferíveis, determinadas por sua estrutura biológica e história particular, mas que, concomitantemente, permanece em contínua interação com os outros sujeitos em diversos domínios de ação, operando emocionalmente e na linguagem [16].

O usuário aprendiz tem o papel de aprender algo vinculado às suas intenções para se engajar em uma comunidade ou grupo, em que existe algo a ser aprendido. O que implica percorrer um processo social e ter disponibilidade para aprender habilidades, capacidades, procedimentos, resolução de problemas, que caracterizam o meio e alimentam no participante 0 sentido de pertencimento nele [18].

O engajamento ocorreu no fórum de forma processual, sendo esse da periferia para o centro de um ou outro. Os participantes iniciaram o fórum respondendo às perguntas propostas, e à medida que o engajamento foi ocorrendo as trocas ocorreram entre aluno-aluno. Cabe ressaltar que, dentre o total de participantes ativos no fórum, a maioria $(56 \%)$ das participações foram classificadas pelo In map Moodle como interatividade alta a média, cada participante postou mais de três mensagens no fórum.

Dessa forma, aprender corresponde ao processo de passagem da condição de novato, de recém-chegado a uma determinada comunidade ou grupo, em que se encontrem determinados saberes, para a condição de experiente em uma dada situação ou contexto desse meio, sobretudo pelo engajamento em atividades reais [18].

Observa-se que o aprendizado proposto através do fórum pela premissa do conhecimento e da experiência individual foi capaz de causar perturbações, trocas e a renegociação continuada dos significados e, então, pelo relacionamento social o aprendizado tornou-se partilhado em comunidade.

A aprendizagem está baseada na experiência e nos relacionamentos sociais. $O$ aprendizado pode ser definido como um realinhamento de experiências e de competências, um puxando o outro, transformando as identidades dos membros da comunidade, que são construídas nas relações sociais. A identificação de um indivíduo com a comunidade acontece e depende da capacidade dessa comunidade em negociar seus próprios significados, respeitando a participação e a não participação, o engajamento e o distanciamento. São valorizados aspectos como a contribuição ativa do indivíduo na comunidade em detrimento das relações de poder, a capacidade do indivíduo e da comunidade em construir processos de orientação, de reflexão e de exploração de novos contextos de troca e aprendizagem, os conflitos são vistos como pontos que determinam a eficácia das ações sociais da comunidade [15].

Outro fator importante foi a multidisciplinaridade presente no fórum por diversas categorias profissionais da área da saúde. A multidisciplinaridade auxiliou na construção social para propor soluções comuns para um dado problema inicial. Na prática clínica, considera-se a necessidade de a equipe de saúde trabalhar de forma compartilhada no sentido da articulação das ações. As ações de saúde articuladas requerem que os profissionais reconheçam e coloquem em evidência as conexões e os nexos existentes entre as intervenções realizadas, aquelas referidas ao seu próprio processo de trabalho e as ações executadas pelos demais integrantes da equipe [19].

Os estudantes conseguiram através do diálogo e interatividade realizar um direcionamento comum, respeitando as individualidades de cada profissão, para a resolutividade 
do problema inicialmente posto no fórum. Na equipe multidisciplinar, os integrantes da equipe devem buscar o melhor caminho, que consiga agregar os diversos conhecimentos disponíveis nessa atmosfera de trabalho, respeitando as individualidades e fazendo uso das diferenças como algo favorável no processo que envolve a busca da resolutividade dos conflitos da população pela qual são responsáveis [20]. Dessa forma, o caráter multidisciplinar teve potencial criador e resolutivo para a construção social, e a Cognição Situada maximiza esse construto.

A informática e seus recursos apresentam um novo paradigma na educação, no qual o uso de tecnologias possibilita e auxilia no processo didático, por meio de recursos interativos capazes de estimular o raciocínio crítico-reflexivo, e não apenas meramente pelo uso do computador.

O desenvolvimento de um fórum deve ser minuciosamente estruturado e embasado em teorias de aprendizagem, que ofereçam suporte aos objetivos e às metas previstas na proposta educacional. Neste estudo, a utilização da Teoria da Cognição Situada, alicerçada no princípio da utilização dos problemas como ponto de partida para a aquisição e integração de novos conhecimentos, foi instigada pelo processo de aprendizado centrado no participante.

A Teoria da Cognição Situada é uma base teórica que se mostrou consistente para o processo de ensino-aprendizagem proposto neste estudo, por considerar o aluno/participante como um ser social interativo, capaz de estabelecer relações e de produzir um conhecimento interdisciplinar contextualizado em sua realidade prática.

Em relação ao fórum, foi possível perceber que à medida que os participantes entrecruzavam informações de forma colaborativa e dinâmica, no empenho de articular, incitar e responder a discussão, o fórum de discussão passou a ser tido como contexto dialógico e de intercognição. É fundamental a presença do outro, o envolvimento mútuo, para motivar as discussões e legitimar a construção do conhecimento colaborativo.

Dessa forma é possível afirmar que o fórum permitiu consolidar e ampliar o conhecimento a respeito do tema a partir da experiência prévia dos participantes, das reflexões sobre a prática profissional e da proposta de ações intervencionistas. O estímulo à discussão entre tutor-aluno e aluno-aluno, possibilita a consolidação do conhecimento acerca do tema estudado.

Cabe destacar, ainda, a necessidade dos docentes de se apropriarem educacionalmente desses novos métodos de EaD para transformar a prática no ensino superior, ainda tão marcada por paradigmas de uma formação cartesiana, bancária e limitada.

\section{Referências}

1. Lave J. Cognition in practice: mind, mathematics, and culture in everyday life. Cambridge: University Press; 1988.

2. Clancey WJ. Situated cognition: on human knowledge and computer representations. Cambridge: University Press, 1997.

3. Costa DAS, Silva RF, Lima VV, Ribeiro ECO. Diretrizes curriculares nacionais das profissões da Saúde 2001-2004: análise à luz das teorias de desenvolvimento curricular. Interface 2018;22(67):1183-95. https://doi.org/10.1590/1807-57622017.0376

4. Oliveira DAMR, Melo VL, Duarte EAS, Sá SPC, Andrade M, Cavalcanti ACD. Atuação do tutor no ensino superior a distância: revisão integrativa. Rev Enferm UFPE on line 2014;8(4):1029-37. https://doi.org/10.5205/reuol.5829-50065-1-ED-1.0804201431

5. Festas MIF. A aprendizagem contextualizada: análise dos seus fundamentos e práticas pedagógicas. Educ Pesqui 2015;41(3):713-27. https://doi.org/10.1590/S15179702201507128518

6. Ruiz-Moreno L, Leite MTM, Ajzen C. Formação didático-pedagógica em saúde: habilidades cognitivas desenvolvidas pelos pós-graduandos no ambiente virtual de aprendizagem. Ciênc Educ 2013;19(1):217-29. https://doi.org/10.1590/S151673132013000100015

7. Bicalho RNM, Oliveira MCSL. O processo dialógico de construção do conhecimento em fóruns de discussão. Interface 2012;16(41):469-84. https://doi.org/10.1590/S141432832012005000028

8. Minayo MCS. O desafio do conhecimento: pesquisa qualitativa em saúde. 14 ed. São Paulo: Hucitec; 2014. 
9. Barrenechea CA. Cognição situada e a cultura da aprendizagem: algumas considerações. Educar 2000;1(16):139-53. https://doi.org/10.1590/0104-4060.211

10. Bittencourt IM, Mercado LPL. Evasão nos cursos na modalidade de educação a distância: estudo de caso do Curso Piloto de Administração da UFAL/UAB. Ensaio: Aval Pol Públ Educ 2014;22(83):465-504.

11. Associação Brasileira de Educação a Distância. Censo EAD BR: Relatório Analítico da Aprendizagem a Distância no Brasil. Ibpex, Curitiba; 2018.

12. Silva GJ, Maciel DA. A presença docente do professor-tutor online como suporte à autonomia do estudante. Psicologia da Educação 2014;38:35-48.

13. Cescon E. Cognição situada e aprendizagem em contextos escolares. Itinerario Educativo 2016;68:37-50. https://doi.org/10.21500/01212753.2946

14. Lemos GC, Almeida LS. Compreender, raciocinar e resolver problemas: Novo instrumento de avaliação cognitiva. Análise Psicológica 2019;37(2):119-33. https://doi.org/10.14417/ap.1583

15. Wenger E. Communities of practice: learning, meaning and identify. New York: Cambridge Press; 1998.

16. Venancio LS, Borges MEN. Cognição situada: fundamentos e relações com a Ciência da Informação. Enc Bibli: R Eletr Bibliotecon Ci Inf 2006;18(22):30-37.

17. Zilio D, Carrara K. Mentalismo e explicação do comportamento: aspectos da crítica behaviorista radical à ciência cognitiva. Acta Comportamentalia 2008;16(3):399-417.

18. Oliveira RG, Di Giorgi CAG. Princípios da cognição situada e as diretrizes curriculares nacionais para a formação de professores. Educ 2011;34(3):360-8.

19. Peduzzi M. Trabalho em equipe de saúde no horizonte normativo da integralidade, do cuidado e da democratização das relações de trabalho. In: Pinheiro R, Mattos RA, Barros MEB. Rio de Janeiro: IMS/UERJ, CEPESC; ABRASCO; 2007.

20. Silva MVS, Miranda GBN, Andrade MA. Sentidos atribuídos à integralidade: entre o que é preconizado e vivido na equipe multidisciplinar. Interface 2017;21 (62):589-99. http://dx.doi.org/10.1590/1807-57622016.0420 